\title{
Microwave Ablation in Intermediate Hepatocellular Carcinoma in Cirrhosis: An Italian Multicenter Prospective Study
}

\author{
Antonio Giorgio ${ }^{1,2}$, Pietro Gatti ${ }^{3}$, Luca Montesarchio ${ }^{1}$, Maria Gabriella Merola ${ }^{6}$, \\ Ferdinando Amendola ${ }^{1}$, Andrea Calvanese ${ }^{1}$, Gaetano Iaquinto ${ }^{5}$, Massimiliano Fontana ${ }^{5}$, \\ Emanuela Ciraci ${ }^{3}$, Stefano Semeraro ${ }^{3}$, Bruno Santoro ${ }^{6}$, Carmine Coppola ${ }^{4}$, Paolo Matteucci ${ }^{7}$ \\ and Valentina Giorgio*8
}

\begin{abstract}
${ }^{1}$ Department of Internal Medicine, Interventional Ultrasound Unit, Tortorella Clinical Hospital, Salerno, Italy; ${ }^{2}$ Department of Surgery, Interventional Ultrasound Unit, Ruesch Clinical Institute, Naples, Italy; ${ }^{3}$ Department of Internal Medicine, Interventional Ultrasound Unit, Ostuni Hospital, Ostuni, Italy; ${ }^{4}$ Department of Internal Medicine, Hepatology Interventional Unit, Gragnano Hospital, Gragnano, Italy; ${ }^{5}$ Interventional Ultrasound Unit, S. Rita Medical-Surgical Hospital, Atripalda, Italy; ${ }^{6}$ Interventional Ultrasound Unit, Athena Clinical Institute, Piedimonte, Italy; ${ }^{7}$ Department of Radiation Oncology, Campus Biomedico University, Rome, Italy; ${ }^{8}$ Department of Pediatrics, Fondazione Policlinico Universitario A. Gemelli, Università Cattolica del Sacro Cuore,
\end{abstract} Rome, Italy

\begin{abstract}
Background and Aims: To report long-term results in treatment of intermediate hepatocellular carcinoma (HCC) in cirrhotics using new high-powered microwaves (MWS) ablation alone. Methods: This multicenter study included 215 cirrhotics (age range: 67-84 years; 137 males; 149 Child A, 66 Child B) who underwent percutaneous ultrasound-guided high-powered MWS ablation instead of transarterial chemoembolization. Among the patient population, 109 had a single nodule $(\varnothing 5.3-8 \mathrm{~cm})$ [group A], 70 had 2 nodules $(\varnothing 3-6 \mathrm{~cm}$ ) [group B] and 36 had 3-5 nodules $(\varnothing 1.5-6.8 \mathrm{~cm}$ ) [group C]. MWS ablation efficacy was evaluated using enhancedcomputed tomography and/or magnetic resonance imaging. Primary end-point was 5-year cumulative overall survival (OS). Results: On enhanced-computed tomography and/or magnetic resonance imaging, complete ablation rates were $100 \%$ for $1.5-3.5 \mathrm{~cm}$ nodules. In nodules $>3.5-5 \mathrm{~cm}$, it was $89 \%$ for the first ablation and $100 \%$ for the second. For lesions $>5-8 \mathrm{~cm}$, ablation was up to $92 \%$. Overall, $1-$, 3- and 5-year survival rates were 89,60 , and $21 \%$, respectively. The cumulative OS rate of group A was $89 \%, 66 \%$ and $34 \%$ at 1,3 and 5 years. The cumulative OS rate of group B was $88 \%, 60 \%$ and $11 \%$ at 1,3 and 5 years. The cumulative OS rate of group $C$ was $86 \%, 55 \%$ and $0 \%$. The 5 -year survival rate was significantly different among the groups $(p<0.001)$. One patient died from rupture of HCC. Upon multivariate analysis, preablation total bilirubin $>1.5 \mathrm{mg} / \mathrm{dL}$ was an independent factor for predicting
\end{abstract}

Keywords: Hepatocellular carcinoma; Intermediate stage; Microwaves ablation; Cirrhosis; HCC.

Abbreviations: AFP, alpha-fetoprotein; BCLC, Barcelona Clinic Liver Cancer; CEUS, contrast-enhanced ultrasound; DFS, disease-free survival; HCC, hepatocellular carcinoma; ITA.LI.CA, Italian Liver Cancer; mRECIST, Modified Response Evaluation Criteria in Solid Tumors; MWS, microwaves; OS, overall 5-year survival rate; PFS, progression-free survival; PT, prothrombin time; RF, radiofrequency; TACE, transarterial chemoembolization; US, ultrasound.

Received: 22 February 2018; Revised: 4 April 2018; Accepted: 16 April 2018

*Correspondence to: Antonio Giorgio, Tortorella Clinical Hospital, Salerno 80131, Italy. Tel: +39-081-248-3198, E-mail: agiorgio28@gmail.com lower survival. Conclusions: Percutaneous MWS ablation of intermediate HCC is safe and effective in inducing large volume of necrosis in intermediate HCC nodules, providing long-term survival rates similar to transarterial chemoembolization. Preablation total bilirubin $>1.5 \mathrm{mg} / \mathrm{dL}$ as expression of liver function reserve is the main factor predicting a worse outcome.

Citation of this article: Giorgio A, Gatti P, Montesarchio L, Merola MG, Amendola F, Calvanese A, et al. Microwave ablation in intermediate hepatocellular carcinoma in cirrhosis: An Italian multicenter prospective study. J Clin Transl Hepatol 2018; 6(3):251-257. doi: 10.14218/JCTH.2018.00013.

\section{Introduction}

Liver cancer is the second most frequent cause of cancer-related death worldwide, and the sixth most frequent cancer. Moreover, it has been estimated that both hepatocellular carcinoma (HCC) incidence and mortality are increasing worldwide. ${ }^{1,2}$ The Barcelona Clinic Liver Cancer (BCLC) is one of the most widely adopted staging systems, and considers the characteristics of the neoplasm, underlying liver disease and performance status. $^{3}$ In the BCLC algorithm, the tumor is classified as early, intermediate and advanced. Intermediate-stage is defined as extensive multifocal disease without vascular invasion in patients with preserved liver function and absence of cancer-related symptoms. ${ }^{3}$ The standard treatment of intermediate stage of $\mathrm{HCC}$ is based on transarterial chemoembolization (TACE). No other treatment options are considered in this algorithm. ${ }^{3}$

In the last years, percutaneous ablation techniques have gained great popularity in treatment of very-early and early HCC, for which they are considered first-line therapy. ${ }^{4-6}$ Since the introduction of percutaneous ethanol injection in 1986, ${ }^{7}$ new and effective thermal ablation modalities have been introduced, such as radiofrequency (RF) and microwaves (MWS). Compared to RF, MWS ablation has emerged as a promising technique for larger ablation areas and faster ablation rates. ${ }^{8}$

MWS ablation induces coagulative necrosis through the application of electromagnetic waves through the antenna 
Giorgio A. et al: HP-MWs ablation in intermediate HCC-cirrhosis

(i.e. microwave probe). Dielectric hysteresis resulting in kinetic energy is the mechanism by which tissue (water molecules) is heated. ${ }^{9}$ MWS ablation offers several advantages over other forms of thermal ablation, ${ }^{10}$ such as high thermal efficiency resulting in larger ablation volumes, a higher capability of coagulating blood vessels and a faster ablation time. Another important advantage of MWS over RF ablation is a less severe heat sink effect, ${ }^{10}$ that is one of the main limitations of $\mathrm{RF}^{8}$ Due to such advantages over RF ablation, larger volumes of necrosis can be induced by MWS and, as a consequence, larger HCC nodules can be ablated. Introduction of new devices and new MWS generators to the market has allowed for the development and application of high-powered antennas (at $2450 \mathrm{MHz}$ ) and new more powerful generators (operating up to $200 \mathrm{~W}$ ), leading to very large volume of necrosis and increasing the sphericity of the necrosis area. 8,11

In fact, using this new technique, an over $80 \%$ complete ablation rate has been reported in HCC nodules up to $8 \mathrm{~cm}$ in diameter. $^{11}$

The aim of our multicenter, prospective study was to report long-term results in treatment of intermediate $\mathrm{HCC}$ in cirrhotic patients using new high-powered MWS alone for percutaneous ablation under ultrasound (US) guidance. The primary end-point was to evaluate cumulative overall 5-year survival (OS), and the secondary end-points were the rates of radiological necrosis, ${ }^{12}$ disease-free survival (DFS) and progression-free survival (PFS).

\section{Methods}

The following hospitals from Southern Italy participated in the study: Tortorella Clinical Hospital, Salerno; Ruesch Clinical Institute, Naples; Ostuni Hospital, Ostuni; Gragnano Hospital, Gragnano; S. Rita Medical- Surgical Hospital, Atripalda; Athena Clinical Institute, Piedimonte.

From May 2011 to April 2016, 277 cirrhotic patients with intermediate HCC were consecutively seen in our Institutions and were prospectively included into the study. All patients had clinical, biochemical and imaging findings of cirrhosis. For all patients, evidence of HCC nodules was seen for the first time on US abdominal examination mainly, and no patient was ever treated before. Prior to enrollment, all patients underwent routine blood tests, including alpha-fetoprotein (AFP) measurement, ${ }^{13}$ US abdominal examination, contrast-enhanced (CE)US using Sonovue (Bracco Imaging Italia, Milano, Italy) and enhanced computed tomography (eCT) and/or enhanced magnetic resonance (eMRI).

Stage of intermediate HCC was assessed with eCT and/or eMRI in all cases. All 277 cirrhotics were asked to undergo percutaneous US-guided MWS ablation alone, instead of TACE for treatment of their HCC nodules. In the six institutions, all 277 patients were informed that TACE was still considered the standard-of-care for treatment of their intermediate HCC; at the same time, characteristics of percutaneous ablation using MWS were clearly explained to all 277 consecutive patients. Following receipt of this information, 62 patients did not accept participation in the study, while 215 patients (age range: $67-$ 84 years, mean: 73 years) accepted participation and underwent MWS ablation alone instead of TACE (forming the study group). The majority of patients had HCV-related cirrhosis, which is prevalent in our geographic area. ${ }^{14}$ Table 1 reports the clinical and laboratory characteristics of our series.

Diagnosis of HCC was made according to the American Association for the Study of Liver Diseases practice guidelines ${ }^{15}$
Table 1. Characteristics of our series

\begin{tabular}{ll}
\hline Characteristic & Value \\
\hline Age in years & $73 \pm 5$ \\
Male sex & $137(63.7 \%)$ \\
Hepatitis C virus & $157(73.0 \%)$ \\
Hepatitis B virus & $58(27.0)$ \\
Child A & $149(69.3)$ \\
Child B & $66(30.7)$ \\
Diameter of nodules in cm & $5.9 \pm 0.7$ \\
Number of nodules & $2.3 \pm 0.7$ \\
Total bilirubin in $\mathrm{mg} / \mathrm{dL}$ & $1.6 \pm 0.4$ \\
Prothrombin time as \% & $67 \pm 0.149$ \\
Platelet count in $\mathrm{mm}^{3}$ & $81000 \pm 8350$ \\
AFP in $\mathrm{ng} / \mathrm{dL}$ & $0.49 \pm 114$ \\
Albumin in $\mathrm{g} / \mathrm{dL}$ & $2.9 \pm 1.2$ \\
\hline
\end{tabular}

Data presented as $n(\%)$ or mean \pm SD.

and staging of intermediate HCC was based on the BCLC algorithm. ${ }^{3}$ Inclusion criteria were as follows: a) not eligible for surgery (resection or liver transplantation) or refusal of surgery; b) single nodule $>5 \mathrm{~cm}$ and no larger than $8 \mathrm{~cm}$; c) two or more nodules (up to 5) with at least one $>5 \mathrm{~cm}$ and no larger than $7 \mathrm{~cm}$; d) platelet count $>50,000 \mathrm{~mm}^{3}$; e) prothrombin time (PT) not less than 50\%; and f) total bilirubin $<=2.5 \mathrm{mg} / \mathrm{dL}$. Exclusion criteria were as follows: a) ascites; b) thrombosis of the portal venous system; and c) extrahepatic spread of the disease.

Among the study population, 109 patients had a single nodule $(\varnothing 5.3-8 \mathrm{~cm}$, mean: $6.4 \mathrm{~cm}$ ) [group A], 70 had 2 nodules ( $\varnothing 3-6 \mathrm{~cm}$, mean: $5.8 \mathrm{~cm}$ ) [group B] and 36 had 3-5 nodules $(\varnothing 1.5-6.8 \mathrm{~cm}$, mean: $6.1 \mathrm{~cm}$ ) [group C]. Table 2 reports the numbers and diameters of the nodules of the three groups.

Percutaneous ablation was performed under US guidance using a high-powered MWS device (Acculis MTA System, Microsulis Medical Ltd, Hampshire, UK) with a single antenna (at $2450 \mathrm{MHz}$ ) connected with a powerful generator (working from $60 \mathrm{~W}$ to $140 \mathrm{~W}$ ). The applicator comprised a $1.8-\mathrm{mm}$ diameter stainless steel shaft tipped with a ceramic trocar array. The applicator was cooled with sterile saline and contained a thermocouple to monitor shaft temperature during use.

In all cases, only one antenna was used per session for ablation. For nodules $1.5-3 \mathrm{~cm}$, only one US-guided percutaneous insertion was performed. For $3-5 \mathrm{~cm}$ nodules, one to two insertions were performed in the same session. For nodules $>5 \mathrm{~cm}$, up to 3 insertions were performed in the

Table 2. Number of patients, number and diameter of nodules

\begin{tabular}{llll}
\hline Group & Patients & Nodules & Nodules diameter in $\mathrm{cm}$ \\
\hline A & 109 & 1 & $6.4(5.3-8.0)$ \\
B & 70 & 2 & $5.8(3.0-6.0)$ \\
C & 36 & $3-5$ & $6.1(1.5-6.8)$ \\
\hline
\end{tabular}

Data are presented as $n$ or median (range). 
same session. When more than one insertion was performed, the antenna was withdrawn from the liver and reinserted percutaneously in a different untreated portion of the nodule. The approach was the same for nodules located in the dome or capsular location. In subcapsular located lesions, the antenna was inserted through at least $1 \mathrm{~cm}$ of liver parenchyma between the liver surface and the margin of the nodule, when possible. If this was not possible, superficially located lesions were directly punctured.

In all cases, CEUS was carried out with Sonovue ${ }^{\circledR}(2.4 \mathrm{~mL})$ as follows: a) before ablation (to evaluate the entire hypervascular pattern of HCC nodules); b) during ablation (to detect any hypovascular area induced by ablation and the remnant arterial enhanced tissue still to-be treated); c) at the end of procedure. Ablation procedures were performed under unconscious sedation, with fentanyl as induction and Propofol as maintenance, as reported elsewhere. ${ }^{16}$

The ablation sessions were scheduled as follows: 1-2 sessions in group A patients; $2-3$ sessions in group $B$ patients; and up to 4 sessions in group $C$ patients. In all cases, at the beginning of the ablation procedure, the power of the MWS generator was set at $100 \mathrm{~W}$, and during the procedure it was increased up to 120-140 W, according to the homogeneous (or non-) distribution of hyperechogenicity within the nodule. During the procedure, when multiple insertions were performed in the same session, the hyperechoic artifact was handled by using CEUS to distinguish hypervascular (untreated) zones by hypovascular (necrotic) areas induced by ablation. CEUS was also used to visualize the distal margin of the nodule to verify that it also appeared necrotic (i.e. hypoechoic on CEUS imaging). In this manner, CEUS was also used to verify if the attempt to obtain an ablated margin was successful.

Track ablation was used in all patients at the end of procedures when antenna was withdrawn from the nodule (i.e. the generator was set at $100 \mathrm{~W}$ for 1 minute and then the antenna was withdrawn). The day after the MWS ablation, all patients underwent clinical, biochemical and abdominal US examinations and they were discharged if no complication was noted. Patients were followed-up every 2 months with laboratory tests, including AFP and abdominal US examination. Efficacy of MWS ablation was evaluated using CEUS, eCT and/ or eMRI at 1 month after the end of the scheduled protocols. ${ }^{12}$ According to the Modified Response Evaluation Criteria in Solid Tumors (mRECIST) evaluation system, in all treated nodules, complete radiological ablation of nodule/s was defined as absence of arterial enhancement covering the entire area of the tumor. ${ }^{17}$ The volume of the entire hypovascular ablated area was also noted to verify increased volume with respect to prior ablation volume.

Discrimination between reactive hyperemia and residual peripheral active tumor was assessed on eCT and/or eMRI in the late and equilibrium phases (hyperemia did not present with wash-out, unlike the active residual tumor margin). Complete radiological necrosis was defined as absence of arterial enhanced tissue on CEUS, eCT and /or MRI in any area of the treated nodule at 1 month after the end of the scheduled protocols, i.e. after 1-2 sessions in group A patients, 2-3 sessions in group $B$ patients and up to 4 sessions in group $C$ patients (see later Results section). Incomplete necrosis was defined as presence of arterial enhanced tissue within the treated nodule. In case of incomplete ablation, repeated MWS ablation procedures were performed to achieve complete radiological necrosis of the nodule/s. In these cases, when the 1 month efficacy evaluation showed incomplete radiological necrosis, a new ablation procedure was immediately performed. In patients who underwent more than one session, all previously treated nodules were reevaluated with CEUS during the procedure and lesions that presented with incomplete necrosis on CEUS were retreated using MWS.

The ethical committee of Ostuni Hospital approved the study on behalf of all the other institutions and all patients gave their informed written consent.

\section{Statistical analysis}

Values were expressed as mean +/- SD or median. Cumulative OS was estimated by the Kaplan-Meier method, and statistical comparison of survival distribution was analyzed by the logrank test. Eleven possible factors affecting survival were analyzed (i.e. age, sex, Child's class of cirrhosis, total bilirubin level, albumin, PT, platelet count, AFP, number of nodules, diameter of nodules and number of antenna insertions). Associations with a $p$-value $\leq 0.1$ upon univariate analysis were entered into a Cox's stepwise multivariate regression analysis. A $p$-value $<0.05$ in a two-tailed test was considered statistically significant. Statistical analysis was performed using the SPSS 15.0 statistical software (SPSS Inc., Chicago, IL, USA).

\section{Results}

\section{Radiological necrosis rates and survival}

All patients but one (see below) were treated according to the scheduled protocol. A total of 792 sessions were performed; the number of insertions ranged from 1 to 2 (mean: 1.7 ) in group A patients, from 2 to 3 (mean: 2.4 ) in group B patients and from 3 to 4 (mean: 3.7 ) in group $C$ patients. The total number of antenna insertions was 1847 . The hospital stay ranged from 1 to 3 days (mean: 1.7 days). After the MWS ablation procedures, according to eCT and /or eMRI findings, the complete ablation rates were $100 \%$ for $1.5-3.5 \mathrm{~cm}$ nodules. For $>3.5-5 \mathrm{~cm}$ lesions, a complete radiological ablation was observed in $89 \%$ of cases after the first scheduled ablation session and $100 \%$ after the second scheduled ablation session. In lesions $>5-8 \mathrm{~cm}$, complete radiological ablation was observed in $74 \%$ of cases after the first ablation session and in $92 \%$ of cases after the last scheduled ablation session. The follow-up ranged from 7 to 67 months (median: 37 months).

The 1-, 2-, 3-, 4- and 5- year cumulative OS rates were $89 \%, 81 \%, 60 \%, 40 \%$ and $21 \%$, respectively. The cumulative OS rates for group A were $89 \%, 66 \%$ and $34 \%$ at 1,3 and 5 years, respectively, for group B were $88 \%, 60 \%$ and $11 \%$ at 1 , 3 and 5 years, respectively and for group C were $86 \%, 55 \%$ and $0 \%$, respectively. The 5 -year survival was significantly different among the three groups $(p<0.001)$ (Fig. 1). DFS rates were $90 \%, 65 \%$ and $36 \%$ for group $A, 85 \%, 64 \%$ and $15 \%$ for group $B$, and $82 \%, 44 \%$ and $0 \%$ for group $C$ at 1,3 and 5 years, respectively. There was a statistically significant difference regarding 5-year DFS among the three groups ( $p$ $<0.001$ ) and regarding 3 -year DFS between groups A and B versus group $\mathrm{C}(p<0.001)$ (Fig. 2$)$.

The 1-, 3- and 5-year PFS rates were $90 \%, 83 \%$ and $82 \%$ for group $A, 64 \%, 62 \%$ and $50 \%$ for group B and $40 \%, 21 \%$ and $17 \%$ for group $C$, respectively, with a statistical significant difference between the three groups $(p<0.001)$ (Fig. 2). Local recurrences were observed in $29.2 \%$ of cases and distant 


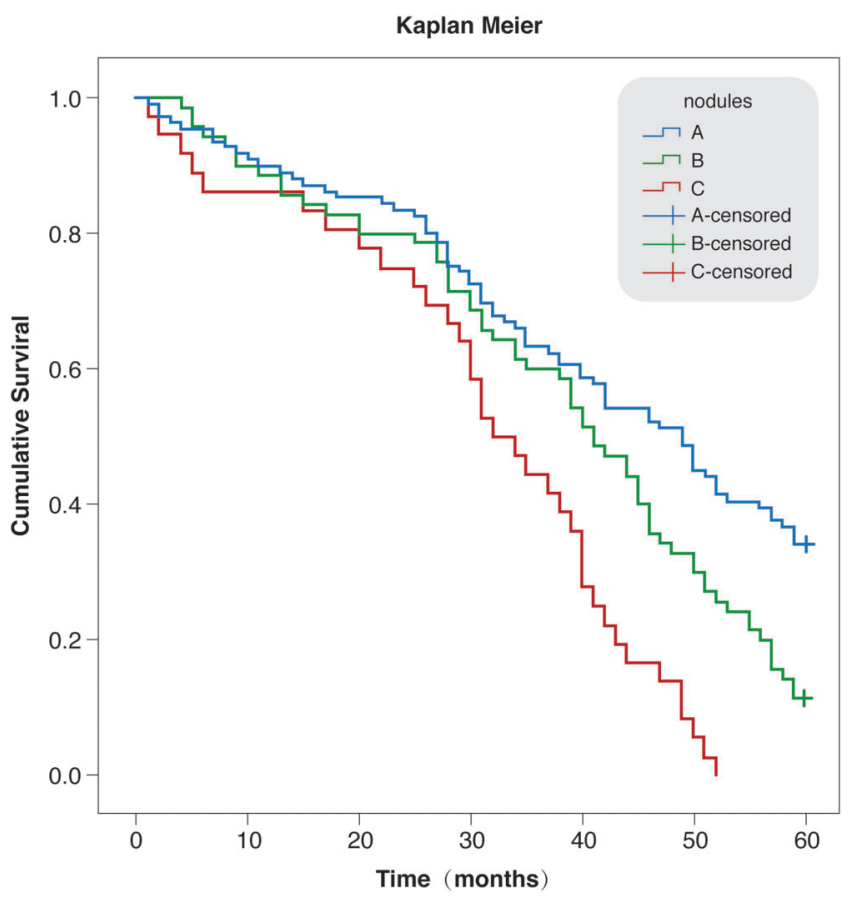

Fig. 1. Overall cumulative 5-years survival of the three groups of our series.

recurrences in $16.6 \%$, and both were treated using MWS ablation. Extrahepatic spread occurred in $3.6 \%$ of cases.

Upon univariate analysis, the number of nodules, diameter of nodules, AFP and preablation total bilirubin $>1.5 \mathrm{mg} / \mathrm{dL}$ were independent factors affecting survival. Upon multivariate analysis, only preablation total bilirubin $>1.5 \mathrm{mg} / \mathrm{dL}$ was the independent factor predicting survival (Table 3 ).

\section{Complications}

One Child A patient (female, age 80 years) with a single $6-\mathrm{cm}$ $\mathrm{HCC}$ nodule located in the VI segment ( 2 antenna insertions in a single session) died from rupture of the HCC nodule with hemoperitoneum and shock 20 hours after the MWS ablation. Only nine patients presented with mild or moderate ascites 2-7 days after MWS ablation, which disappeared with diuretics therapy within 4-9 days. Abdominal pain was observed soon after procedure in $29(10.5 \%)$ patients, treated with analgesic drugs (diclofenac or tramadol), and $32(11.5 \%)$ patients presented with mild to moderate fever, treated with paracetamol. No other major or minor complications were observed. Except for the patient who died, no other patient required blood transfusion. No hemothorax or pneumothorax was observed. During the follow-up, no case of seeding was observed.

\section{Discussion}

In our study, percutaneous high-powered MWS ablation alone was demonstrated to be able to induce up to $89 \%$ radiologic necrosis after the first and $100 \%$ after the second ablation session in 3-5 cm HCC lesions, while the rate of radiological necrosis was observed in up to $92 \%$ of cases in $5-8 \mathrm{~cm} \mathrm{HCC}$ nodules at the end of the scheduled sessions. Moreover, cumulative OS rates at 1,3 , and 5 years were $89 \%, 60 \%$

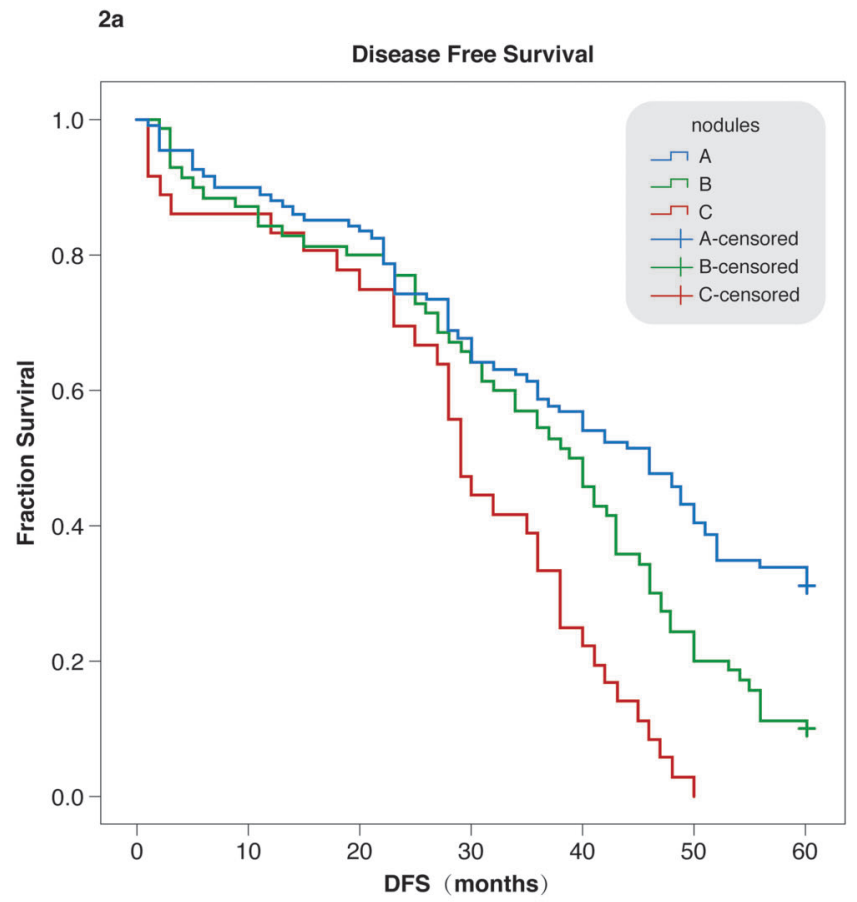

2b

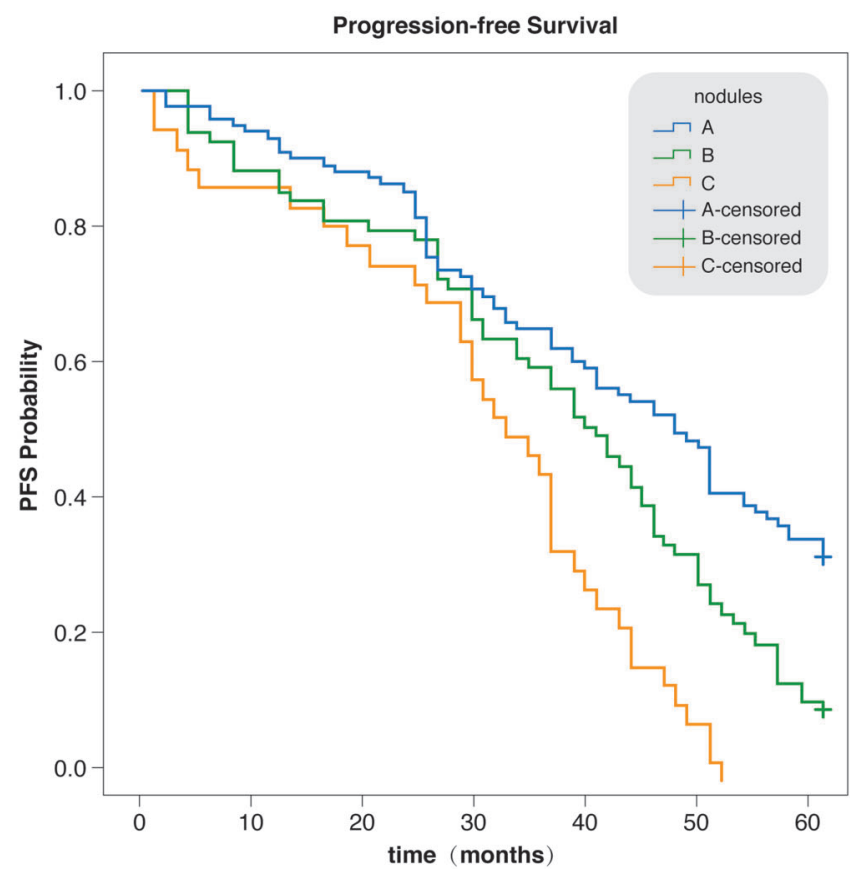

Fig. 2. Survival rates. 5-year disease-free survival (A) and 5-year progressionfree survival (B) of the three groups of our series.

and $21 \%$, respectively. Furthermore, cumulative 5 -year survival was significantly reduced in group $C$ patients and DFS of this group had decreased already at 3 years with respect to patients in both groups $A$ and $B$, indicating that the number of nodules may have a major role in affecting DFS. Similar results were observed analyzing the curves of PFS, with 3-year PFS being statistically lower in the group $C$ patients with respect to the other groups. Again, such rates of PFS 
Giorgio A. et al: HP-MWs ablation in intermediate HCC-cirrhosis

Table 3. Univariate and multivariate analyses of prognostic factors for cumulative overall survival

\begin{tabular}{|c|c|c|c|c|}
\hline \multirow[b]{2}{*}{ Significant variable } & \multicolumn{2}{|l|}{ Univariate analysis } & \multicolumn{2}{|l|}{ Multivariate analysis } \\
\hline & OR $(95 \% C I)$ & $p$ & OR $(95 \% C I)$ & $p$ \\
\hline Age in years: $<65$ vs. $>65$ & $1.127(0.742-1.739)$ & 0.847 & & \\
\hline Male sex: yes/no & $0.963(0.527-1.785)$ & 0.985 & & \\
\hline Child-Pugh class: A vs. B & $0.997(0.843-2.781)$ & 0.952 & & \\
\hline PT value as $\%: \leq 60 \%$ vs. $>60 \%$ & $0.989(0.754-1.341)$ & 0.987 & & \\
\hline Platelet count: $<60.000 / L$ vs. $>60.000 / L$ & $1.058(0.555-2.069)$ & 0.847 & & \\
\hline Total bilirubin in mg/dL: $\leq 1.5$ vs. $>1.5$ & $3.016(1.629-5.589)$ & 0.003 & $1.546(1.872-2.686)$ & 0.021 \\
\hline Albumin in $\mathrm{g} / \mathrm{L}:<3$ vs. $>3$ & $0.989(0.754-1.311)$ & 0.986 & & \\
\hline AFP in $\mu \mathrm{g} / \mathrm{L}:<20$ vs. $>20$ & $1.157(0.755-1.736)$ & 0.03 & & \\
\hline Number of nodules: 1 vs. $>1$ & $1.39(0.793-1.732)$ & 0.031 & & \\
\hline Diameter of nodules in $\mathrm{cm}:<5$ vs. $>5$ & $1.164(0.754-1.763)$ & 0.04 & & \\
\hline Number of needle insertions: 1 vs. $>1$ & $2.874(1.891-4.457)$ & 0.018 & & \\
\hline
\end{tabular}

Abbreviations: AFP, alpha-fetoprotein; CI, confidence interval; OR, odds ratio; PT prothrombin time.

suggest that the diameter of the nodules is important in predicting the outcome of patients with large nodules. The univariate analysis showed that the diameter of the nodules was an independent factor affecting survival, together with the number of nodules, AFP and pre-ablation total bilirubin. Finally, upon multivariate analysis, only preprocedure total bilirubin $>1.5 \mathrm{mg} / \mathrm{dL}$ was the main predictor of survival. These results clearly indicate that, at the end, the residual function hepatic reserve is the main factor to predict success prior to performing MWS ablation in such patients.

Our results are in keeping with the recent literature data. Medhat et al. ${ }^{18}$ showed that complete ablation was achieved in $19 / 26(73.1 \%)$ of patients with $5-\mathrm{cm}$ to $7-\mathrm{cm}$ HCC lesions. Zhang and coworkers ${ }^{11}$ treated 45 patients with $60 \mathrm{HCC}$ lesions, and obtained $82.61 \%$ necrosis at first ablation in $3-5 \mathrm{~cm}$ lesions and $100 \%$ at second ablation. Moreover, in $5-\mathrm{cm}$ to $8-\mathrm{cm}$ HCC lesions they obtained complete ablation in $64.29 \%$ at first ablation and $85.71 \%$ at second ablation. ${ }^{18}$ Both studies concluded that microwave ablation by the percutaneous approach is safe and effective in the treatment of large HCC tumors. ${ }^{11,18}$

Up to now, many treatment options are available in the scientific scenario for management of HCC in cirrhosis. If surgery (resection and transplantation) and ablation are considered curative treatments for HCC in the stage A of the BCLC algorithm as well as TACE in the stage $B,{ }^{3,15}$ other options are now available in treatment of HCC. These options include oral sorafenib ${ }^{19}$ and $Y 90 .{ }^{20} \mathrm{Up}$ to now, sorafenib has been recommended only in the advanced stage (BCLC stage C), ${ }^{3}$ while results of $Y 90$ still need to be validated and, anyway, all the presented studies have been performed in veryadvanced disease cases. Surgery is recommended for treatment of intermediate HCC only in the Hong Kong Score System ${ }^{21}$ and not in the American Association for Study of Liver Diseases and European Association for the Study of the Liver practice guidelines that continue to consider only TACE for treatment of intermediate $\mathrm{HCC}^{3,15}$ But, very recent data do not support these Western recommendations. ${ }^{22-24}$ Evaluating the role of surgery to be expanded beyond the BCLC algorithm, ${ }^{22}$ these recent data demonstrated that surgical resection had better OS than TACE for patients with intermediate stage HCC, without any significant increase in postoperative complication or 30-day mortality rates. ${ }^{22-24}$

Anyway, given the current Western recommendations and considering that patients included in our study were not candidates for surgery, in our opinion the best comparison should be made between ablation and TACE. In our study, the 5 -year cumulative OS rates are similar if not better to longterm results regarding cumulative survivals for intermediate HCC using TACE alone or combined with other modalities. ${ }^{24-26}$ In fact, recent studies have reported $38 \%, 15 \%$ and $12 \%$ OS at 1,3 and 5 years, respectively, with TACE alone ${ }^{23}$ and $94 \%$, $52 \%$ and $26.1 \%$ at 1,3 and 5 years when combined with radiofrequency ablation. ${ }^{25}$ Many intraarterial therapies are available for treatment of HCC, such as chemoembolization, bland embolization or drug-eluting beads-TACE. But, up to now, all these procedures have showed similar efficacy. ${ }^{26-29}$

Obviously, our statement that MWS ablation and TACE have similar efficacy in treatment of intermediate HCC deserves caution because our study was not carried out in a randomized controlled manner. Nevertheless, in our opinion, some considerations can be made. Firstly, many criticisms have been raised about the possibility that a single technique can be valid in all different circumstances since the definition of intermediate $\mathrm{HCC}$ in the BCLC algorithm refers to a large variety of number and diameter of neoplastic nodules, and TACE is the only technique that is considered as the goldstandard for treatment. Recently, Bolondi and co-workers, ${ }^{30}$ using a new subclassification of intermediate HCC (B1 to B4 stages), proposed ablation in addition to TACE in the B1stage. Although in our study we did not use Bolondi's subclassification, our results go exactly in this direction. Secondly, Farinati et al. ${ }^{31}$ on the basis of the Italian Liver Cancer (known as ITA. LI.CA) cohort of $3027 \mathrm{HCC}$ cases that was recruited from 1986 to 2008 by the ITA.LI.CA group, concluded that in clinical practice, TACE cannot be considered the best approach for BCLC stage $B$ patients who represent a heterogeneous population and are often suitable for more aggressive therapies, which lead to a better survival. After evaluating 485 consecutive BCLC-B patients from the ITA.LI.CA database retrospectively, it was concluded that in "everyday practice TACE represents the first-line therapy in a half of patients with naïve BCLC-B HCC 
since treatment choice is driven not only by liver function and nodule characteristics, but also by contraindications to procedures, comorbidities, age and patient opinion". ${ }^{32,33}$ Therefore the conclusion of Shiand and $\mathrm{Zhai}^{34}$ seems reasonable. In their recent review on advance in Image-Guided Locoregional Therapy for Hepatocellular Carcinoma, they stated that "combination treatment modalities, such as thermal-based ablation combined with TACE or ${ }^{125}$ I seed implant brachytherapy, may further broaden their clinical indications for $\mathrm{HCC}^{\prime \prime}{ }^{34}$

Our study has some limitations. The main limitation is the absence of a controlled group of patients treated with TACE. Therefore, a randomized controlled trial evaluating the outcome of intermediate HCC patients treated with MWS ablation versus TACE is needed. But, researchers should be aware that strict criteria comparing the two groups should be adopted in the selection of patients, considering that the definition of intermediate $\mathrm{HCC}$ in the BCLC algorithm refers to a large variety of the number and diameter of neoplastic nodules. The same is for the absence of a comparison with surgical resection, especially for large, single nodules in selected patients. But, as said before, our patients were not candidates for surgery. A second limitation is the use of a single-applicator in our MWS ablation procedures. We are well aware that multiple applicators can create larger ablation zones. But, in our routine work in interventional ultrasound, the long-time experience of the operators involved in the study makes the difference. In fact, using high-powered MWS under US guidance, in few instants, can also induce large nodules to become rapidly hyperechoic just like when ethanol injection was used in treating large HCC nodules with the socalled one-shot technique. ${ }^{16,35}$ On the other hand, as far as complications are concerned, in our experience only one bleeding event occurred with 1847 punctures. It is conceivable that when using multiple applicators, the risk of bleeding would be doubled or tripled.

In conclusion, in our experience, percutaneous US-guided MWS ablation alone for treatment of intermediate HCC in cirrhosis was safe and effective in inducing large-volume necrosis in HCC nodules and it can obtain appreciable cumulative 5-year survival.

\section{Conflict of interest}

The authors have no conflict of interests related to this publication.

\section{Author contributions}

Guarantor of integrity of entire study, study concepts, study design (AG), data acquisition and data analysis/interpretations, manuscript final version approval (AG, PG, LM, MGM, FA, $A C, G I, M F, E C, S S, B S, C C, P M, V G)$, manuscript drafting or manuscript revision for important intellectual content (AG, VG), statistical analysis (LM), manuscript editing ( $A G, V G, P M)$.

\section{References}

[1] Ferlay J, Soerjomataram I, Dikshit R, Eser S, Mathers C, Rebelo M, et al. Cancer incidence and mortality worldwide: sources, methods and major patterns in GLOBOCAN 2012. Int J Cancer 2015;136:E359-E386. doi: 10. 1002/ijc. 29210.

[2] Lafaro KJ, Demirjian AN, Pawlik TM. Epidemiology of hepatocellular carcinoma. Surg Oncol Clin N Am 2015;24:1-17. doi: 10.1016/j.soc.2014.09.001.
[3] Bruix J, Reig M, Sherman M. Evidence-based diagnosis, staging, and treatment of patients with hepatocellular carcinoma. Gastroenterology 2016;150: 835-853. doi: 10.1053/j.gastro.2015.12.041.

[4] N'Kontchou G, Mahamoudi A, Aout M, Ganne-Carrié N, Grando V, Coderc E, et al. Radiofrequency ablation of hepatocellular carcinoma: long-term results and prognostic factors in 235 Western patients with cirrhosis. Hepatology 2009;50:1475-1483. doi: 10.1002/hep.23181.

[5] Xu Y, Shen Q, Wang N, Wu PP, Huang B, Kuang M, et al. Microwave ablation is as effective as radiofrequency ablation for very-early-stage hepatocellular carcinoma. Chin J Cancer 2017;36:14. doi: 10.1186/s40880-017-0183-x.

[6] Facciorusso A, Serviddio G, Muscatiello N. Local ablative treatments for hepatocellular carcinoma: An updated review. World J Gastrointest Pharmacol Ther 2016;7:477-489. doi: 10.4292/wjgpt.v7.i4.477.

[7] Livraghi T, Festi D, Monti F, Salmi A, Vettori C. US-guided percutaneous alcohol injection of small hepatic and abdominal tumors. Radiology 1986; 161:309-312. doi: 10.1148/radiology.161.2.3020612.

[8] Poggi G, Montagna B, DI Cesare P, Riva G, Bernardo G, Mazzucco M, et al. Microwave ablation of hepatocellular carcinoma using a new percutaneous device: preliminary results. Anticancer Res 2013;33:1221-1227.

[9] Lubner MG, Brace CL, Hinshaw JL, Lee FT Jr. Microwave tumor ablation: mechanism of action, clinical results, and devices. J Vasc Interv Radiol 2010;21:S192-S203. doi: 10.1016/j.jvir.2010.04.007.

[10] Liang $P$, Wang $Y$. Microwave ablation of hepatocellular carcinoma. Oncology 2007;72:124-131. doi: 10.1159/000111718.

[11] Zhang NN, Lu W, Cheng XJ, Liu JY, Zhou YH, Li F. High-powered microwave ablation of larger hepatocellular carcinoma: evaluation of recurrence rate and factors related to recurrence. Clin Radiol 2015;70:1237-1243. doi: 10.1016/j.crad.2015.06.092.

[12] Ahmed M, Solbiati L, Brace CL, Breen DJ, Callstrom MR, Charboneau JW, et al. Image-guided tumor ablation: standardization of terminology and reporting criteria-a 10-year update. J Vasc Interv Radiol 2014;25:1691-1705.e4. doi: $10.1016 / j$.jvir.2014.08.027.

[13] Riaz A, Ryu RK, Kulik LM, Mulcahy MF, Lewandowski RJ, Minocha J, et al. Alpha-fetoprotein response after locoregional therapy for hepatocellular carcinoma: oncologic marker of radiologic response, progression, and survival. J Clin Oncol 2009;27:5734-5742. doi: 10.1200/JCO.2009.23.1282.

[14] ITACAN: Cancer in Italy, Version 2.0. Available from: http://itacan.ispo. toscana.it/English/itacan.htm, accessed January 31, 2014.

[15] Bruix J, Sherman M. Management of hepatocellular carcinoma: an update. Hepatology 2011;53:1020-1022. doi: 10.1002/hep.24199.

[16] Giorgio A, Tarantino L, Francica G, Mariniello N, Nuzzo A, del Viscovo L, et al. One-shot percutaneous ethanol injection of liver tumors under general anesthesia: preliminary data on efficacy and complications. Cardiovasc Intervent Radiol 1996;19:27-31.

[17] Lencioni R, Llovet JM. Modified RECIST (mRECIST) assessment for hepatocellular carcinoma. Semin Liver Dis 2010;30:52-60. doi: 10.1055/s-00301247132.

[18] Medhat E, Abdel Aziz A, Nabeel M, Elbaz T, Zakaria Z, Shousha H, et al. Value of microwave ablation in treatment of large lesions of hepatocellular carcinoma. J Dig Dis 2015;16:456-463. doi: 10.1111/1751-2980.12259.

[19] Llovet JM, Ricci S, Mazzaferro V, Hilgard P, Gane E, Blanc JF, et al. Sorafenib in advanced hepatocellular carcinoma. N Engl J Med 2008;359:378-390. doi: 10.1056/NEJMoa0708857.

[20] Edeline J, Gilabert M, Garin E, Boucher E, Raoul JL. Yttrium-90 microsphere radioembolization for hepatocellular carcinoma. Liver Cancer 2015;4:16-25. doi: 10.1159/000343878.

[21] Yau T, Tang VY, Yao TJ, Fan ST, Lo CM, Poon RT. Development of Hong Kong Liver Cancer staging system with treatment stratification for patients with hepatocellular carcinoma. Gastroenterology $2014 ; 146: 1691-1700 . e 3$. doi: 10.1053/j.gastro.2014.02.032.

[22] Hyun $\mathrm{MH}$, Lee YS, Kim JH, Lee CU, Jung YK, Seo YS, et al. Hepatic resection compared to chemoembolization in intermediate to advanced stage hepatocellular carcinoma: a meta-analysis of high-quality studies. Hepatology 2018. doi: 10.1002/hep.29883.

[23] Liang $\mathrm{L}$, Xing $\mathrm{H}$, Zhang $\mathrm{H}$, Zhong J, Li C, Lau WY, et al. Surgical resection versus transarterial chemoembolization for BCLC intermediate stage hepatocellular carcinoma: a systematic review and meta-analysis. HPB (Oxford) 2018;20:110-119. doi: 10.1016/j.hpb.2017.10.004.

[24] Zhao YN, Zhang YQ, Ye JZ, Liu X, Yang HZ, Cong FY, et al. Hepatic resection versus transarterial chemoembolization for patients with Barcelona Clinic Liver Cancer intermediate stage Child-Pugh A hepatocellular carcinoma. Exp Ther Med 2016;12:3813-3819. doi: 10.3892/etm.2016.3810.

[25] Xu RC, Liu HC, Li JL, Li K, Ou SY, Yu ZY, et al. Long-term outcome of transcatheter arterial chemoembolization after radiofrequency ablation as a combined therapy for Chinese patients with hepatocellular carcinoma. Curr Med Res Opin 2015;31:1553-1560. doi: 10.1185/03007995.2015.1058249.

[26] Golfieri R, Giampalma E, Renzulli M, Cioni R, Bargellini I, Bartolozzi C, et al. Randomised controlled trial of doxorubicin-eluting beads vs conventional chemoembolisation for hepatocellular carcinoma. Br J Cancer 2014;111: 255-264. doi: 10.1038/bjc.2014.199. 
[27] Facciorusso A, Bellanti F, Villani R, Salvatore V, Muscatiello N, Piscaglia F, et al. Transarterial chemoembolization vs bland embolization in hepatocellular carcinoma: A meta-analysis of randomized trials. United European Gastroenterol J 2017;5:511-518. doi: 10.1177/2050640616673516.

[28] Nakamura A, Osonoi T, Terauchi Y. Relationship between urinary sodium excretion and pioglitazone-induced edema. J Diabetes Investig 2010;1: 208-211. doi: 10.1111/j.2040-1124.2010.00046.x.

[29] Massani M, Stecca T, Ruffolo C, Bassi N. Should we routinely use DEBTACE for unresectable HCC? CTACE versus DEBTACE: a single-center survival analysis. Updates Surg 2017;69:67-73. doi: 10.1007/s13304-017-0414-3.

[30] Bolondi L, Burroughs A, Dufour JF, Galle PR, Mazzaferro V, Piscaglia F, et al. Heterogeneity of patients with intermediate (BCLC B) Hepatocellular Carcinoma: proposal for a subclassification to facilitate treatment decisions. Semin Liver Dis 2012;32:348-359. doi: 10.1055/s-0032-1329906.

[31] Farinati F, Vanin V, Giacomin A, Pozzan C, Cillo U, Vitale A, et al. BCLC stage $B$ hepatocellular carcinoma and transcatheter arterial chemoembolization: a 20-year survey by the Italian Liver Cancer group. Liver Int 2015;35: 223-231. doi: 10.1111/liv.12649.

[32] Giannini EG, Moscatelli A, Pellegatta G, Vitale A, Farinati F, Ciccarese F, et al. Application of the intermediate-stage subclassification to patients with untreated hepatocellular carcinoma. Am J Gastroenterol 2016;111:70-77. doi: 10.1038/ajg.2015.389.

[33] Pecorelli A, Lenzi B, Gramenzi A, Garuti F, Farinati F, Giannini EG, et al. Curative therapies are superior to standard of care (transarterial chemoembolization) for intermediate stage hepatocellular carcinoma. Liver Int 2017;37: 423-433. doi: 10.1111/liv.13242.

[34] Shi Y, Zhai B. A recent advance in image-guided locoregional therapy for hepatocellular carcinoma. Gastrointest Tumors 2016;3:90-102. doi: 10.1159/ 000445888.

[35] Giorgio A, Tarantino L, de Stefano G, Perrotta A, Aloisio V, del Viscovo L, et al. Ultrasound-guided percutaneous ethanol injection under general anesthesia for the treatment of hepatocellular carcinoma on cirrhosis: long-term results in 268 patients. Eur J Ultrasound 2000;12:145-154. 